\title{
Reviewers for Kew Bulletin Volume 67
}

We are grateful to the following people who reviewed articles for Kew Bulletin during the years 2011 - 2012.
D. Albach
F. Adema
C. Archer
D. Bailey
A. Barfod
M. Blanco
L. Bohs
S. Breckle
B. Bremer
M. Callmander
F. de D. Calonge
P. Cantino
M. Celis
B. Conn
T. Daniel
S. Dressler
L. Dreyer
M. de la Estrella
A. Ford
S. Fuse
S. Gale
M. G. Gilbert
A. M. Giulietti
D. Harris
T. Hodkinson
B. K. Holst
P. Hovenkamp
J. Iganci
P. Kessler
C. Kiel
M. Koch
B. Kropp
R. Kynast
P. P. Lowry III
V. Malécot
J. Manning
A. Meerow
U. Meve
D. Middleton
J. Morawetz
A. M. Muasya
H. Nagamasu
M. Newman
H. P. Nooteboom
J. Paiva
J. Parnell
C. Pendry
P. Phillipson
J. Pipoly
A. P. Prata
J. Pruski
R. Rice
R. Rutishauser
M.-S. Samian
L. G. Saw
M. Sobral
G. Staples
P. Stevens
M. Strong
T. Stützel
S. Suddee
P. Suksathan
M. Tamura
E. Tripp
L. J. G. van der Maesen
J. F. Veldkamp
J. White
P. Wilkie
C. Williams
J. R. I. Wood
S. Zona 